\title{
Estrategia de sostenibilidad para reservas naturales privadas. Estudios de caso en Costa Rica
}

Sustainability strategy for private nature reserves. Case studies in Costa Rica

Estratégia de sustentabilidade para reservas naturais privadas. Estudos de caso na Costa Rica

María Margarita Arrieta García / Universidad de Costa Rica, Universidad de la Costa, Colombia/ marrieta18@cuc.edu.co Michael Moya Calderón / Universidad de Costa Rica, Costa Rica / michael.moya c@ucr.ac.cr

Recibido: $17 / 11 / 2020$

Aceptado: $7 / 2 / 2021$

Publicado: $15 / 6 / 2021$

\section{RESUMEN}

En Costa Rica existen más de 210 reservas naturales privadas miembros de la Asociación Red Costarricense de Reservas Naturales, y otras no vinculadas a esta organización; sin embargo, se conoce poco sobre ellas y sus aportes a la conservación. El objetivo de esta investigación fue proponer un modelo de estrategia de sostenibilidad para reservas naturales privadas, con base en el caso del cantón San Ramón, Alajuela; que aporte al fortalecimiento de los procesos de conservación por medio de la evaluación de los componentes ambiental, sociocultural, económico y administrativo. Para ello fueron seleccionadas cuatro reservas naturales privadas de dicho cantón, a partir de criterios previamente establecidos; y se desarrolló un sistema de 28 indicadores de sostenibilidad para reservas privadas, el cual se aplicó a las mismas. La reserva Parque Aventura San Luis obtuvo el mayor nivel de sostenibilidad (71.5\%), mientras que la reserva Cerro La Tinajita presentó el más bajo (43.3\%). En las cuatro reservas, la dimensión sociocultural mostró los mejores niveles de sostenibilidad y la dimensión económica los más bajos. Teniendo en cuenta los resultados de la evaluación, se propone un plan estratégico de sostenibilidad.

Palabras clave: áreas protegidas privadas, conservación de áreas protegidas, desarrollo sostenible, ecoturismo, indicadores de sostenibilidad 


\begin{abstract}
In Costa Rica there are more than 210 private nature reserves members of the Costa Rican Association of Natural Reserves and others that are not linked to this organization; however, little is known about these and their contributions to conservation. The study aimed to propose a sustainability strategy model for private natural reserves, based on the case of San Ramón, Alajuela; that will contribute to the strengthening of conservation processes, through the assessment of its environmental, sociocultural, economic and administrative components. Four private nature reserves of the canton were selected, based on previously established criteria. Initially, a system of 28 sustainability indicators for private reserves was developed, this system was applied in the four participating reserves. Parque Aventura San Luis reserve obtained the highest level of sustainability ( $71.5 \%)$; meanwhile, the lowest level was obtained by Cerro La Tinajita reserve (43.3\%). In general terms, the sociocultural dimension was the one that presented the best levels of sustainability and the economic dimension obtained the lowest levels when analyzing the four reserves. Finally, a strategic sustainability plan was proposed, based on the results obtained in the assessment.
\end{abstract}

Keywords: conservation of protected areas, ecotourism, private protected areas, sustainability indicators sustainable development

\title{
RESUMO
}

Na Costa Rica existem mais de 210 reservas naturais privadas que são membros da Associação Costarriquenha de Reservas Naturais, além de outras não vinculadas a esta organização; entretanto, pouco se sabe sobre elas e as suas contribuições para a conservação. Esta pesquisa teve como objetivo propor um modelo de estratégia de sustentabilidade para reservas naturais privadas, baseado no caso do cantão San Ramón, Alajuela; que contribua para o fortalecimento dos processos de conservação através da avaliação das componentes: ambiental, sociocultural, econômico e administrativo. Quatro reservas naturais privadas do cantão foram selecionadas com base em critérios previamente estabelecidos. Inicialmente foi desenvolvido um sistema de 28 indicadores de sustentabilidade para reservas privadas, o qual foi aplicado nas quatro reservas participantes. Os resultados indicaram que a reserva Parque Aventura San Luis obteve o maior nível de sustentabilidade (71.5\%), enquanto o nível mais baixo foi obtido pela reserva Cerro La Tinajita (43.3\%). Em termos gerais, a dimensão sociocultural foi a que apresentou os melhores níveis de sustentabilidade e a dimensão econômica os níveis mais baixos. Tendo em vista os resultados da avaliação, se propõe um plano estratégico de sustentabilidade.

Palavras chave: áreas protegidas privadas, conservação de áreas protegidas, desenvolvimento sustentável, ecoturismo, indicadores de sustentabilidade 


\section{INTRODUCCIÓN}

Costa Rica constituye una de las naciones más ricas en biodiversidad por unidad de área en el planeta, razón por la que ha sido catalogada como un hotspot de biodiversidad, junto a los demás países de Mesoamérica (de Albuquerque et al., 2015). Desde principios de la década de 1990, ha experimentado un proceso de recuperación de su extensión forestal, debido a la creación de incentivos para apoyar el manejo de sus bosques naturales, como el Certificado de Protección de Bosques, el cual evolucionó al pago por servicios ambientales en 1997 (de Camino et al., 2000). Dichas iniciativas perseguían revertir las estadísticas negativas que se observaban en el país entre 1960 y 1986, cuando la cobertura forestal pasó de $59.5 \%$ a $40.8 \%$; y sus puestas en vigor, junto a otras medidas ambientales, propiciaron que los valores ascendieran de $40.8 \%$ a $51.4 \%$ en el período comprendido entre 1986 y 2010 (Sánchez-Azofeifa, 2015).

Desde entonces, el bosque se ha considerado como un componente clave para el alcance de los objetivos ambientales del país, además de constituir uno de los atractivos más populares de la nación como destino turístico, pues la belleza escénica que estos exhiben al mantener sus ecosistemas con una amplia diversidad de flora y fauna, le ha merecido tal reconocimiento a nivel internacional (Banco Central de Costa Rica, 2016).

En relación con lo anterior, entre 2010 y 2018 en Costa Rica se evidencia un aumento del $1 \%$ en su cobertura boscosa; sin embargo, aunque alrededor del 52.4\% del territorio es de cobertura forestal, solo el $25.4 \%$ se encuentra bajo alguna de las categorías de manejo de Áreas Silvestres Protegidas incluidas en el Sistema Nacional de Áreas de Conservación (Pavlotzky, 2019), o sea, solo cerca de la mitad de los bosques. Singularmente, en los últimos años se ha producido un leve aumento en las áreas protegidas de propiedad privada (Programa Estado de la Nación, 2016), aun cuando estos valores no se correspondan con el estado deseado.

Al respecto, si bien en el país existen más de 210 reservas naturales privadas que forman parte de la Red Costarricense de Reservas Naturales (Barrientos et al., 2019) y otras no vinculadas a esa asociación, resulta importante puntualizar que se conoce muy poco sobre ellas y sus aportes a la conservación.

Como afirman Bingham et al. (2017), las áreas protegidas de propiedad privada se encuentran subrepresentadas en las bases de datos internacionales, a pesar de los avances positivos evidenciados en etapas recientes, y de que la mayoría de los lugares importantes para la biodiversidad se encuentran fuera de las áreas protegidas formales (Dinerstein et al., 2017). En correspondencia, existe una falta de información sobre la existencia de este tipo de espacios a nivel mundial y una muy poca comprensión de los procesos sociales que han llevado a su aumento en el orbe, lo cual reafirma la necesidad de generar guías sobre las políticas y prácticas adecuadas que faciliten la labor que se lleva a cabo en ellas.

El presente artículo se basa en una investigación que tuvo como objetivo 
general, proponer un modelo de estrategia de sostenibilidad para reservas naturales privadas, con base en el caso del cantón San Ramón, Alajuela; que aporte al fortalecimiento de los procesos de conservación de estos espacios por medio de la evaluación de los componentes ambiental, sociocultural, económico y administrativo.

\section{MATERIALES Y MÉTODOS}

\section{Área de estudio}

La investigación se realizó en el cantón San Ramón, provincia Alajuela, Costa Rica, en la región occidental del Valle Central (figura 1); posee una extensión territorial de $1018.6 \mathrm{~km}^{2}$ y está dividido en 14 distritos: San Ramón, Santiago, San Juan, Piedades Norte, Piedades Sur, San Rafael, San Isidro, Ángeles, San
Lorenzo, Alfaro, Volio, Concepción, Zapotal y Peñas Blancas. Más del $50 \%$ de las reservas identificadas y contactadas en el presente estudio se ubican en los distritos Peñas Blancas y Ángeles.

\section{Descripción de la metodología}

Para el desarrollo de la investigación se definieron cuatro fases:

1. Selección de las reservas naturales privadas.

2. Diseño de la herramienta de indicadores de sostenibilidad.

3. Evaluación de la sostenibilidad en las reservas seleccionadas.

4. Formulación de un plan estratégico para la sostenibilidad de las reservas analizadas.

Figura 1. Localización del área de estudio y de las reservas seleccionadas
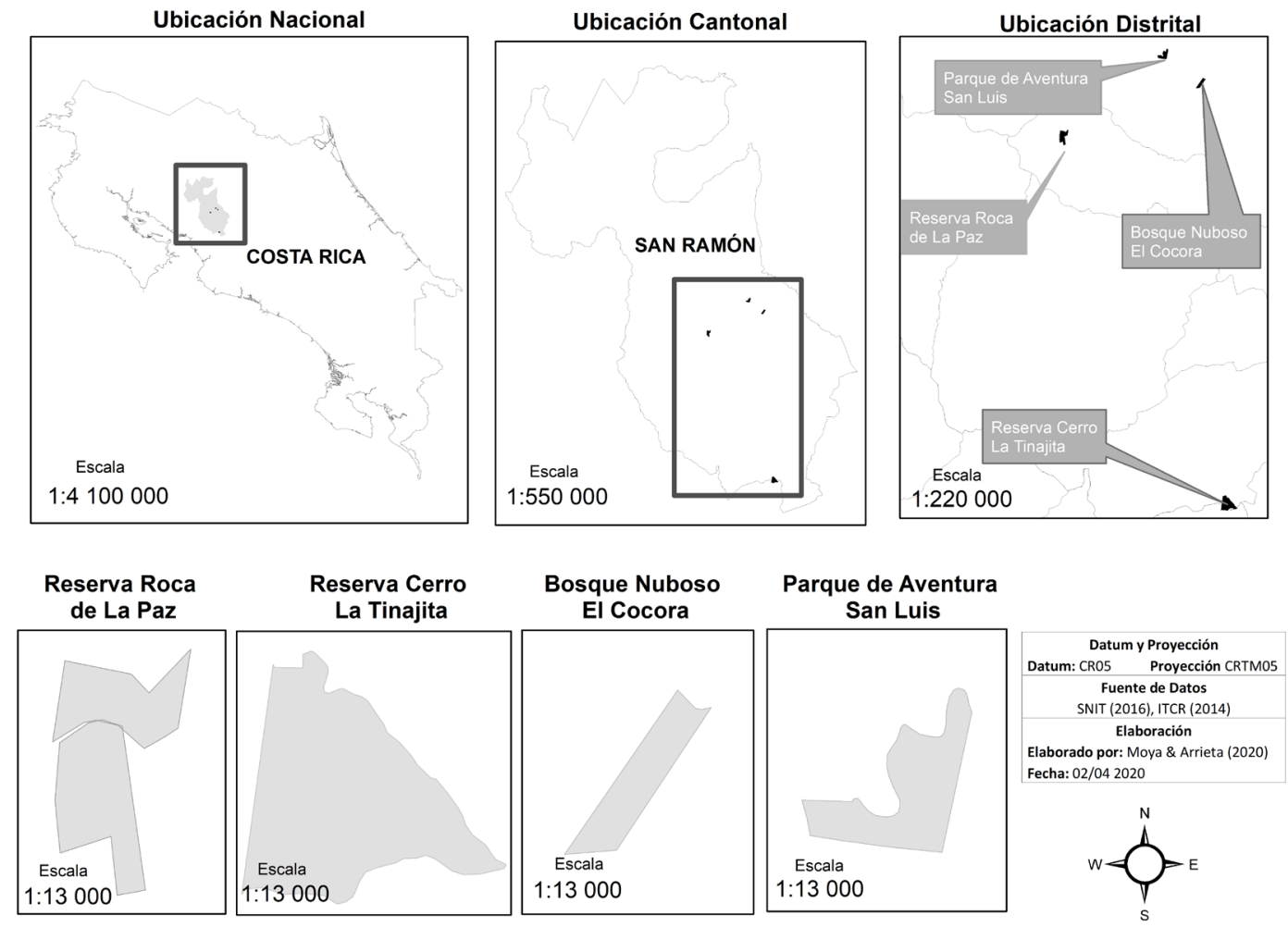

Fuente: Elaboración propia. 


\section{Fase 1. Selección de reservas naturales privadas}

Esta fase se desarrolló entre mayo y noviembre de 2018. En ella se llevaron a cabo tres actividades:

1. Realización del registro de las reservas privadas del cantón San Ramón y su estado actual; para lo cual se efectuaron entrevistas semiestructuradas presenciales y vía correo electrónico a organizaciones como la Red Costarricense de Reservas Naturales y la Fundación Bosque Nuboso de Occidente, con el fin de determinar la situación de la conservación por iniciativa privada en el área de estudio.

2. Solicitud de información sobre las reservas a sus propietarios; ello se realizó a través de un cuestionario estructurado con preguntas abiertas, en el cual se consultaba sobre los siguientes criterios: interés en participar en la investigación, año de creación de la reserva, tipo de propietario, disponibilidad de personal, área en conservación, comunidad más cercana, visitación y accesibilidad. El interés de estos por participar en la investigación y la facilidad de acceso a la información fueron aspectos claves para la selección de las reservas a estudiar. Como es este un estudio de caso, la selección de la muestra fue teórica, teniéndose en cuenta que los casos múltiples son una herramienta poderosa para crear teoría al permitir la replicación y la extensión entre casos individuales. Como no existe una guía del número exacto que debe ser incluido en un estudio de este tipo, los autores de la presente investigación asumen los criterios de Perry (1998), quien plantea que esta decisión se deja al investigador.

3. Selección de las reservas; con este fin, la selección de las cuatro reservas se realizó mediante criterios de mínimos o máximos. Se tuvieron en cuenta el interés en participar en la investigación y la facilidad de acceso a la información como dos ítems individuales, y se plantearon otros siete, para un total de nueve criterios:

- Tiempo: mínimo 10 años dedicados a la conservación.

- Tipo de propietario: la propiedad debe ser individual, familiar, de una asociación u organización no gubernamental (ONG).

- Disponibilidad de personal: por lo menos una persona disponible para el acompañamiento a los investigadores durante el desarrollo del estudio.

- Área en conservación: debe conservar como mínimo 5 ha de bosque.

- Distancia a la comunidad más cercana: no debe exceder los $7 \mathrm{~km}$.

- Visitación: se debe permitir la visita al área, al menos con fines de investigación.

- Accesibilidad:lascondiciones de acceso a la reserva deben ser favorables, ya sea en vehículo particular o facilitado por el propietario.

Para la selección de las cuatro reservas, sobre la base de los nueve criterios establecidos, se planteó una escala nominal dicotómica, 
de la siguiente manera:

- 1: se cumple el criterio

- 0: no se cumple el criterio

Las reservas seleccionadas obtuvieron el puntaje máximo (9). Finalmente, se realizaron visitas de reconocimiento con la finalidad de recorrer las reservas y levantar la información de ubicación por medio del Sistema de Posicionamiento Global.

\section{Fase 2. Diseño de la herramienta de indicadores de sostenibilidad}

Esta fase se desarrolló entre diciembre de 2018 y febrero de 2019. Se diseñó un sistema de indicadores para evaluar la sostenibilidad de las reservas seleccionadas, para lo cual se consideraron los pilares clásicos que la sustentan: ambiental, social (sociocultural), económico y, además, el administrativo.

Los cuatro ámbitos fueron objeto de un análisis preliminar en cada reserva por medio de la observación y la realización de entrevistas semiestructuradas a sus propietarios. Con la aplicación de estas entrevistas se abordaron aspectos como: la tenencia, uso de la propiedad, permanencia, factores que afectarían la duración de la reserva, motivaciones, financiamiento, selección de la ubicación, extensión, incentivos, gestión de la reserva, estrategias de manejo y fortalezas y debilidades; para un total de 34 preguntas abiertas y de opción múltiple.

Por otro lado, se llevó a cabo una revisión bibliográfica para seleccionar los indicadores que se pudieran adaptar e incluir dentro de la herramienta. Lo anterior permitió generar una lista de indicadores previos, los cuales fueron adaptados a la forma de evaluación que proponen Cifuentes et al. (2000), para obtener los indicadores finales de sostenibilidad.

La metodología planteada por Cifuentes et al. (2000), para medir la efectividad en el manejo de áreas protegidas, utiliza una escala de calificación de cinco niveles (0-4); cada uno de ellos se asocia a una ponderación porcentual que expresa el grado de cumplimiento por indicador, que va desde insatisfactorio a muy satisfactorio, como se observa en la tabla 1.

Tabla 1. Escala de calificación y ponderación de indicadores

\begin{tabular}{ccc}
\hline Calificación & \% del óptimo & Significado \\
\hline 4 & $91-100$ & Muy satisfactorio \\
3 & $76-90$ & Satisfactorio \\
2 & $51-75$ & Medianamente satisfactorio \\
1 & $36-50$ & Poco satisfactorio \\
0 & $<35$ & Insatisfactorio \\
\hline
\end{tabular}

Fuente: Adaptada de Cifuentes et al. (2000).

Los indicadores previos se validaron mediante la metodología 3s, propuesta por Cloquell (2003, como se citó en GutiérrezFernández et al., 2012), la cual es una herramienta sencilla y que ha sido empleada en la validación de indicadores, con la participación de diversos actores que buscan reducir la subjetividad de los instrumentos de cuantificación. Esta metodología consiste en tres niveles de validación: 
- Nivel 1. Sui Validatio: la autovalidación se realiza por el investigadoro investigadores que desarrollan el estudio. Su propósito es generar una reflexión interna de lo propuesto.

- Nivel 2. Scienciatis Validatio: la validación científica busca dotar de rigor y objetividad al sistema que ha sido diseñado por el equipo redactor, mediante la integración de los juicios de expertos independientes. Se realizó mediante el método Delphi, en dos rondas. Después de la primera, el cuestionario fue sometido a una prueba de fiabilidad por medio del cálculo del coeficiente Alfa de Cronbach, para comprobar que los criterios (indicadores) y las escalas (opciones de respuesta) propuestos eran consistentes. Este coeficiente se considera bueno si el resultado está entre 0.6 y 0.8 ; mientras que es alto cuando el resultado se encuentra entre 0.8 y 1.0 (Gutiérrez-Fernández et al., 2012).

- Nivel 3. Societatis Validatio: en esta validación social se busca incorporar la participación pública, ya que esto dota al proceso de una mayor transparencia y posibilidad de éxito.

Estos tres niveles de validación son complementarios y a medida que aumenta el nivel, las herramientas adquieren un mayor grado de adecuación para su uso en los estudios particulares donde se apliquen (Gutiérrez-Fernández et al., 2012). Para cada uno de los indicadores finales se diseñó una ficha técnica, en la cual se describe: nombre, definición, finalidad, pertinencia y método de determinación; con su respectiva escala de evaluación.
La clasificación de los indicadores en las cuatro dimensiones del desarrollo sostenible se realizó a criterio de los autores. Inicialmente, se seleccionaron o propusieron 53 indicadores, los cuales fueron sometidos a las siguientes etapas de depuración y al criterio de expertos para su validación por medio del método Delphi.

Para la validación 3s, el nivel 1 se realizó mediante la aplicación de la metodología Crema (recibe su nombre de la primera letra de cada criterio que evalúa) del Banco Mundial, en la cual se calificaron de 1 a 5 los siguientes criterios por indicador: claro, relevante, económico, medible y adecuado (Kuzek y Rist, 2004). En este procedimiento el sistema de indicadores se redujo a 34 .

Se aplicó el método Delphi para el nivel 2 de la validación 3s. Se contactaron 51 posibles expertos en temas de gestión y sostenibilidad de áreas protegidas estatales o privadas, de países como Costa Rica, Cuba, Paraguay, Argentina y Colombia. Únicamente 22 enviaron el formulario para calcular el coeficiente de competencia experta, que es una técnica que se centra en la autovaloración de la persona para determinar su competencia sobre un tema. De estos 22, tres no superaron el puntaje de 0.8; por lo que esta consulta se realizó a los 19 expertos restantes.

Para la ronda 1 del método Delphi se recibieron 14 respuestas. Luego del procesamiento de estas se calculó el coeficiente Alfa de Cronbach, cuyo resultado fue de 0.82 , considerándose la prueba como consistente. La segunda ronda se realizó con estas 14 respuestas y se seleccionaron los indicadores que obtuvieron calificación igual o mayor a 4.0 en promedio, para proceder 
al nivel 3 de la metodología 3s. Se informó a los 14 expertos sobre los resultados de la primera ronda (media) y se les solicitó una nueva respuesta (si estaban o no de acuerdo con los resultados), para la cual, si estaban en desacuerdo, debieron proponer otra calificación. Se obtuvo finalmente la respuesta de 13 expertos. A partir de estos resultados se definieron los ítems que formaron parte de la herramienta estándar de indicadores (tabla 3).

\section{Fase 3. Evaluación de la sostenibilidad en las reservas seleccionadas}

La evaluación fue realizada en cada reserva entre marzo y abril de 2019, mediante la aplicación de la herramienta diseñada en la segunda fase. Cada indicador se valoró siguiendo el método de determinación definido en su ficha técnica, con el fin de obtener el valor final de sostenibilidad presente en aquellas, tal y como se muestra en el anexo 1.

La evaluación se realizó recolectando la información por medio de las visitas al campo y las entrevistas estructuradas (con tres y siete preguntas abiertas sobre cada indicador), estas últimas socializadas previamente a los propietarios. Los valores finales se clasificaron de acuerdo con la tabla 1, con base en los resultados de la evaluación de los indicadores por ámbito.

\section{Fase 4. Formulación de un plan estratégico para el fortalecimiento de la sostenibilidad}

La formulación del plan, su socialización y la devolución de resultados se realizaron entre junio y agosto de 2019. Partiendo de los resultados de la fase tres, se diseñó un plan estratégico para fortalecer la sostenibilidad en las áreas más débiles de las reservas analizadas y, teniendo en cuenta las recomendaciones para la formulación de estrategias, incorporó las prioridades e intereses de conservación aportados por los actores de aquellas.

En este sentido, las prioridades se establecieron de acuerdo con los indicadores que obtuvieron las mediciones más bajas y aquellos que estaban más relacionados con los objetivos de la reserva. Además, se consideraron las capacidades reales del propietario para tomar acciones ante los resultados de la evaluación. De la misma manera, se eligieron como prioritarios aquellos indicadores que al fortalecerse pudieran ayudar a mejorar otros (Arguedas, 2015). Finalmente, se realizó la devolución de los resultados de la investigación a los propietarios de las reservas analizadas, con el fin de que estos conocieran su evaluación de sostenibilidad y las medidas propuestas para el fortalecimiento de la misma.

\section{RESULTADO}

\section{Selección de las reservas naturales privadas}

En la tabla 2 se presentan las 22 reservas contactadas y sus respectivos puntajes de calificación en los criterios establecidos para la selección. El puntaje máximo (9) fue obtenido por las siguientes reservas, al cumplir con todos los criterios:

- Bosque Nuboso El Cocora

- Parque Aventura San Luis

- Roca de La Paz

- Cerro La Tinajita 
Tabla 2. Puntajes de las reservas identificadas para la selección de las participantes en la investigación

\begin{tabular}{|c|c|c|c|c|c|c|c|c|c|c|}
\hline \multirow[b]{2}{*}{ Aspecto } & \multicolumn{9}{|c|}{ Reservas naturales privadas contactadas para la investigación } & \multirow{3}{*}{$\begin{array}{l}\text { Puntaje } \\
\text { final }\end{array}$} \\
\hline & 1 & 2 & 3 & 4 & 5 & 6 & 7 & 8 & 9 & \\
\hline Criterio & $\mathrm{Si}$ & $\mathrm{Si}$ & $\begin{array}{l}>10 \\
\text { años }\end{array}$ & $\begin{array}{l}\text { fam., ind., } \\
\text { asoc. }\end{array}$ & $\begin{array}{c}\geq 1 \\
\text { empleado }\end{array}$ & $\begin{array}{l}\geq 5 \\
\text { ha }\end{array}$ & $\begin{array}{l}\leq 7 \\
\mathrm{~km}\end{array}$ & $\mathrm{Si}$ & $\mathrm{Si}$ & \\
\hline Chachagua Rainforest & \multicolumn{9}{|c|}{ No se obtuvo respuesta } & 0 \\
\hline Rancho Margot & 0 & 0 & 1 & 1 & 1 & 1 & 1 & 1 & 1 & 7 \\
\hline Bosque Nuboso El Cocora & 1 & 1 & 1 & 1 & 1 & 1 & 1 & 1 & 1 & 9 \\
\hline Hotel Villa Blanca & 1 & 0 & 1 & 1 & 1 & 1 & 0 & 1 & 1 & 7 \\
\hline Hotel Tierras Enamoradas & 0 & 0 & 1 & 1 & 1 & 1 & 0 & 1 & 1 & 6 \\
\hline Canopy San Lorenzo & 0 & 0 & 1 & 1 & 1 & 1 & 0 & 1 & 1 & 6 \\
\hline Ecomusas & 1 & 0 & 1 & 1 & 1 & 1 & 1 & 1 & 1 & 8 \\
\hline Finca Luna Nueva & \multicolumn{9}{|c|}{ No se obtuvo respuesta } & 0 \\
\hline Parque Aventura San Luis & 1 & 1 & 1 & 1 & 1 & 1 & 1 & 1 & 1 & 9 \\
\hline Reserva Salto de la Danta & 1 & 1 & 1 & 1 & 0 & 1 & 0 & 1 & 0 & 6 \\
\hline Valle de Los Quetzales & 1 & 1 & 1 & 1 & 0 & 1 & 1 & 1 & 0 & 7 \\
\hline Enlaces No. 1, 2 y 4 & \multicolumn{9}{|c|}{ No se obtuvo respuesta } & 0 \\
\hline Centro Soltis & \multicolumn{9}{|c|}{ No se obtuvo respuesta } & 0 \\
\hline Bosque Nuboso Nectandra & 1 & 0 & 1 & 1 & 1 & 1 & 1 & 1 & 1 & 8 \\
\hline Roca de La Paz & 1 & 1 & 1 & 1 & 1 & 1 & 1 & 1 & 1 & 9 \\
\hline Cerro La Tinajita & 1 & 1 & 1 & 1 & 1 & 1 & 1 & 1 & 1 & 9 \\
\hline $\begin{array}{l}\text { La Calandria Cloudforest } \\
\text { camp }\end{array}$ & 1 & 1 & 1 & 1 & 0 & 1 & 1 & 1 & 0 & 7 \\
\hline Finca Camino Verde & 1 & 1 & 1 & 1 & 0 & 0 & 1 & 0 & 1 & 6 \\
\hline Reserva privada Los Alpes & \multicolumn{9}{|c|}{ No se obtuvo respuesta } & 0 \\
\hline La Tigra Ecolodge & \multicolumn{9}{|c|}{ No se obtuvo respuesta } & 0 \\
\hline
\end{tabular}

Nota: 1. Interés de participación; 2. acceso a la información; 3. tiempo; 4. tipo de propietario (familiar, individual y asociación); 5. disponibilidad de personal; 6. área en conservación; 7. distancia de la comunidad más cercana; 8. visitación; 9. accesibilidad.

Fuente: Elaboración propia.

Definición de indicadores para evaluar de indicadores estándar se sometió a la la sostenibilidad en reservas naturales validación por parte de los propietarios de privadas

El diseño del sistema de indicadores de las cuatro reservas seleccionadas. En cada reserva se excluyeron los indicadores que no sostenibilidad para reservas naturales privadas se presenta en la tabla 3. se consideraron pertinentes de acuerdo con sus características.

En el nivel 3 de la validación 3s, la lista 
Tabla 3. Lista estándar de indicadores de sostenibilidad y sus subindicadores

\section{Dimensión sociocultural}

1. Apoyo y participación comunitaria.

2. Apoyo interinstitucional.

3. Desarrollo de investigaciones.

4. Aplicación de conocimientos tradicionales.

5. Beneficios económicos a las comunidades aledañas.

\section{Dimensión ambiental}

6. Características biogeográficas: conectividad, claridad de límites, cobertura natural.

7. Evaluación de amenazas: amenazas por impactos de la visitación, contaminación, incendios, infraestructura, etc.

8. Usos ilegales: extracción de recursos naturales no renovables o renovables, precarismo, cacería, ganadería, construcción de infraestructura, etc.

9. Manejo de residuos sólidos.

10. Especies amenazadas identificadas.

\section{Dimensión administrativa}

14. Existencia de planes de trabajo.

15. Establecimiento de objetivos de conservación.

16. Personal: motivación, existencia del personal necesario.

17. Infraestructura: interpretación ambiental en la reserva, instalaciones para visitantes, senderos.

18. Leyes y normas generales: claridad y aplicación.

19. Sistema de organización de información.

20. Motivación del propietario para conservar.

21. Aseguramiento del esfuerzo de conservación transgeneracional.

Fuente: Elaboración propia.

Evaluación de la sostenibilidad en las cuatro reservas naturales privadas seleccionadas

La aplicación de la herramienta de indicadores para evaluar la sostenibilidad en las reservas seleccionadas arrojó lo siguientes resultados:

\section{Reserva Natural Bosque Nuboso El Cocora}

De acuerdo con la escala de calificación de la sostenibilidad (Cifuentes et al., 2000), esta reserva se encuentra en un nivel medianamente satisfactorio con un
$55.5 \%$ La dimensión que más le aporta es la sociocultural, con un cumplimiento óptimo del $75 \%$ (figura 2A). Los beneficios económicos a las comunidades aledañas, cobertura natural y claridad de límites en cuanto a las características biogeográficas y la motivación del propietario para conservar fueron indicadores que obtuvieron el máximo puntaje. Por otro lado, la dimensión económica debe ser fortalecida, pues presenta un cumplimiento del $37.5 \%$ con respecto al óptimo (figura $2 A$ ). En este 
sentido, las estrategias que se implementen deben enfocarse en la necesidad de mejorar el manejo presupuestario, para lograr una mejor organización de los ingresos y egresos que recibe la reserva.

Figura 2. Gráfico general de sostenibilidad en las dimensiones evaluadas de cada reserva

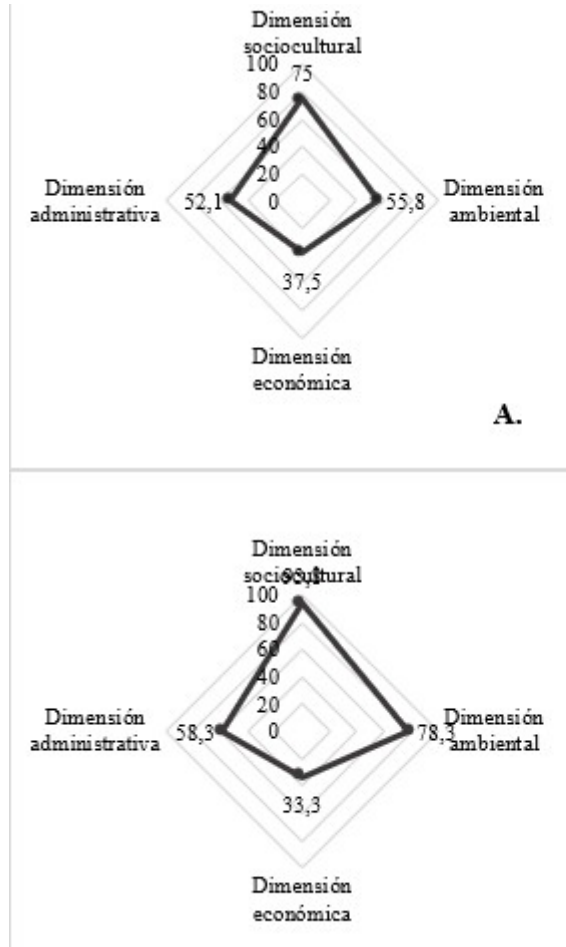

C.

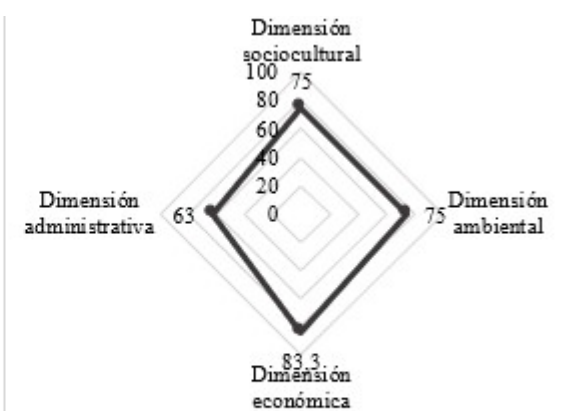

B.

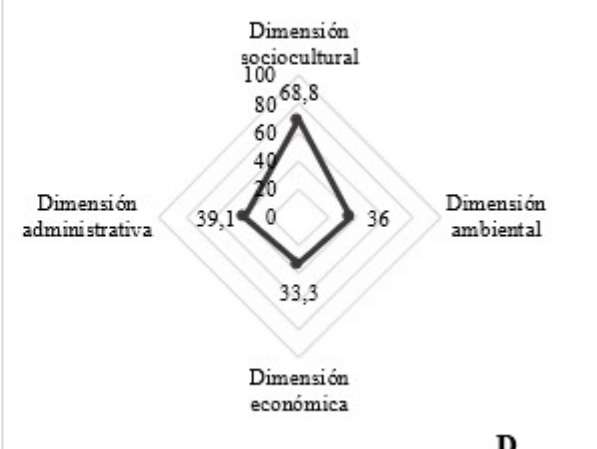

Nota: A. Bosque Nuboso El Cocora, B. Parque Aventura San Luis, C. Roca de La Paz, D. Cerro La Tinajita.

Fuente: Elaboración propia.

\section{Reserva Natural Parque Aventura San Luis}

La sostenibilidad de esta reserva se encuentra en un nivel medianamente satisfactorio (71.5\%), cercano al límite superior, ya que varía entre $51 \%$ y $75 \%$ de cumplimiento. La dimensión que más aporta a la sostenibilidad es la económica, con valores del $83.3 \%$ con respecto al óptimo (figura 2B). En este sentido, algunos de los indicadores que obtuvieron el máximo puntaje son: el financiamiento, apoyo interinstitucional y los beneficios económicos a las comunidades aledañas. La dimensión que requiere mayor fortalecimiento es la administrativa, con un cumplimiento del $63 \%$ con respecto al óptimo (figura 2B). De acuerdo con lo anterior, las estrategias que se implementen deben enfocarse en la interpretación ambiental en la reserva, en cuanto a la infraestructura, la formulación de un plan de trabajo y objetivos de conservación más claros, y para el aseguramiento del esfuerzo de conservación transgeneracional.

\section{Reserva Natural Roca de La Paz}

Su nivel de sostenibilidad es medianamente satisfactorio (66.8\%); sin embargo, el mismo varía entre $51 \%$ y $75 \%$ de cumplimiento, encontrándose más cercana al nivel superior. La dimensión que más aporta a la sostenibilidad es la sociocultural, con 
un cumplimiento del $93.8 \%$ del óptimo (figura 2C). Por su parte, la dimensión económica requiere ser fortalecida, pues presentó un $33.3 \%$ con respecto al óptimo (figura 2C). De acuerdo con lo anterior, las estrategias que se implementen deben enfocarse en la necesidad de mejorar el financiamiento, el manejo presupuestario y la posible aplicación del pago por servicios ambientales.

\section{Reserva Natural Cerro La Tinajita}

El nivel general de sostenibilidad es poco satisfactorio (43.4\%). La dimensión que más le aporta es la sociocultural, con un cumplimiento del $68.8 \%$ del óptimo (figura 2D), donde se destacan como muy satisfactorios, indicadores como el apoyo y la participación comunitaria. Por otro lado, la dimensión que requiere mayor fortalecimiento es la económica, con un cumplimiento del $33.3 \%$ con respecto al óptimo; no obstante, la ambiental (35.8\%) y la administrativa (39.1\%) también requieren acciones urgentes (figura 2D).

\section{Plan estratégico para el fortalecimiento de la sostenibilidad de las reservas naturales privadas}

Las estrategias propuestas para las cuatro reservas analizadas parten de los resultados de su evaluación de sostenibilidad. Estas fueron enviadas a los propietarios de las mismas y se modificaron o complementaron con sus aportes, de tal manera que se adecuaran a sus características y contextos específicos. El plan estratégico diseñado presenta lineamientos generales para mejorar los aspectos menos satisfactorios identificados durante la investigación. Algunas acciones son de implementación individual, mientras que otras requieren del trabajo en equipo de todas las reservas del cantón; por lo cual es importante la aplicación a fondos de organismos internacionales que puedan facilitar el financiamiento de gran parte de ellas. Para este plan estratégico se propusieron cuatro ejes, a partir de los cuales se definieron estrategias, objetivos, acciones, participantes, responsable y resultados esperados.

\section{Eje estratégico 1: investigación y capacitación}

Se enfoca en la investigación y conocimiento de los recursos naturales y servicios ecosistémicos que ofrece cada reserva para implementar acciones que correspondan con su conservación. A su vez, se proponen las capacitaciones necesarias para una mejor gestión y funcionamiento de estas. Lo anterior se planteó al tener en cuenta que el conocimiento que se maneje en la reserva, tanto de los recursos presentes como de los temas que influyen en su funcionamiento, es clave para lograr una gestión más eficiente que conlleve a la consolidación de los procesos de conservación a largo plazo o a perpetuidad.

- Estrategia 1.1. Investigación y conocimiento de los recursos naturales y servicios ecosistémicos presentes en las reservas.

- Estrategia 1.2. Capacitaciones sobre aspectos claves para el manejo de las reservas.

- Estrategia 1.3. Campañas para promover la conservación de vida silvestre y evitar la cacería en las comunidades aledañas

Eje estratégico 2: gestión organizativa y gobernanza

Busca fortalecer la gestión organizativa 
y la gobernanza desde el sector privado. Está enfocado en el fortalecimiento de la participación comunitaria como herramienta fundamental para el buen funcionamiento de las reservas naturales privadas dentro del contexto socioeconómico, donde se desarrollan. De la misma manera, pretende mejorar la sostenibilidad desde el punto de vista financiero, al proponer opciones para obtener financiamiento y una mayor organización a nivel económico, con el fin de que las prioridades de inversión y gestión estén más claras. Finalmente, se busca optimizar la gestión organizativa, tanto de la información que se tiene en torno a la reserva, como de las herramientas de planificación y monitoreo que permitan administrar de manera eficiente los recursos con los que se cuenta.

- Estrategia 2.1. Promoción de la participación comunitaria.

- Estrategia 2.2. Sostenibilidad financiera.

- Estrategia 2.3. Fortalecimiento del sistema de organización.

- Estrategia 2.4. Formulación de planes de trabajo y objetivos de conservación.

\section{Eje estratégico 3: fortalecimiento y mejora de la infraestructura}

Este eje estratégico se enfoca en perfeccionar la infraestructura con la que cuentan las reservas naturales privadas. Propone la implementación de herramientas de interpretación del patrimonio, que permitan una mayor sensibilización de los visitantes con el ecosistema y los recursos presentes en las reservas, para propiciar un mejor entendimiento y cambios de actitudes con respecto a la conservación. También incluye la renovación de los senderos en función de minimizar los riesgos que puedan representar para los visitantes.

- Estrategia 3.1. Implementación de técnicas de interpretación del patrimonio.

- Estrategia 3.2. Mejoramiento de la calidad de los senderos.

\section{Eje estratégico 4: conservación a perpetuidad}

El mismo se enfoca en lograr la consolidación delosprocesos deconservación a perpetuidad en las reservas naturales privadas. Plantea la generación de una propuesta que tribute al reconocimiento formal del Estado hacia dichos espacios, con el fin de que esto les permita acceder a mayores beneficios de asesoría técnica o financiera. También propone la creación de una red cantonal de reservas naturales privadas, que fortalezca las iniciativas de conservación individual por medio del intercambio de experiencias y saberes colectivos; así como la creación de redes de trabajo para la oferta turística colegiada entre los participantes en ellas.

- Estrategia 4.1. Formulación de la propuesta para la creación de categorías de manejo del Sistema Nacional de Áreas de Conservación.

- Estrategia 4.2. Creación de red cantonal de reservas naturales privadas.

\section{DISCUSIÓN}

$\mathrm{Si}$ bien existen antecedentes sobre evaluaciones de la efectividad de manejo en reservas privadas (Pellin y Lima, 2016) o de la efectividad de conservación en las mismas (Shumba et al., 2020), esta es una propuesta contextualizada que permite evaluar la sostenibilidad general en ellas. Sobre este particular, la evaluación de sostenibilidad 
se les ha realizado a distintas categorías de áreas protegidas, pero en la investigación presentada se propone un marco específico, diseñado directamente para las reservas privadas, lo cual es un aporte importante, al ofrecer un primer acercamiento a la aplicación de dichas evaluaciones en este tipo de área protegida.

Los resultados de la primera fase coinciden con lo afirmado por Bingham et al. (2017), ya que las reservas naturales privadas existen en diferentes formas y tamaños; sus propietarios también son diversos (fundaciones, empresas, instituciones religiosas, individuos, entre otros); y sus objetivos de conservación varían entre una y otra. En este sentido, las reservas seleccionadas cuentan con características muy particulares, lo cual permitió darle un enfoque más amplio a la investigación, teniendo en cuenta el tipo de administración, las actividades que se desarrollan en ellas y los ecosistemas que se protegen.

Mientras que Langholz y Lassoie (2001) encontraron que el $40 \%$ de las reservas privadas en Costa Rica realizan ecoturismo raramente o nunca; los resultados de este estudio se contraponen a esa tendencia, pues de las 14 reservas que respondieron en la fase inicial, el $100 \%$ ofrecen servicios ecoturísticos o proyectan hacerlo. Lo anterior coincide con lo hallado por Serenari et al. (2016), quienes manifiestan que las reservas naturales privadas en Chile, al menos las más grandes, aportan al desarrollo sostenible del país a través de esa práctica.

En cuanto a la fase de selección de los indicadores, como aseguran Montaño et al. (2006), la cantidad de indicadores seleccionados para evaluar cualquier proceso debe ser la mínima posible para mantener la mayor certidumbre al menor esfuerzo y costo. Asimismo, Sánchez et al. (2018) también manifiestan que es necesario realizar una selección apropiada de ellos, de acuerdo con los objetivos y recursos disponibles. Además de lo que afirman los autores mencionados, es importante resaltar que tampoco deben presentarse vacíos importantes, por lo que es clave priorizar los aspectos e indicadores indispensables a tener en cuenta. En el presente estudio los indicadores y subindicadores se redujeron de 53 a 28.

Por otro lado, los resultados del diseño del sistema de indicadores guardan relación con lo que plantean GutiérrezFernández et al. (2012), en cuanto a la aplicación de la metodología 3s, ya que se logró dar un mayor grado de adecuación a la herramienta diseñada a medida que aumentó su nivel, es decir, se perfeccionó la priorización de los aspectos para no dejar cuestiones importantes fuera del estudio. La principal ventaja de esta metodología es que permite asegurar que los indicadores propuestos son apropiados e incluyen los aspectos necesarios para poder determinar la sostenibilidad de las reservas naturales privadas.

Por su parte, Stolton y Dudley (2015) afirman que, en muchos casos, el establecimiento y gestión de reservas privadas beneficia a las comunidades locales por la generación de ingresos, empleo y un espacio para el mercado local; ellas permiten el desarrollo de nuevas capacidades de trabajo y otros beneficios indirectos, como las mejoras de la infraestructura. En concordancia, esta investigación encontró que la dimensión 
sociocultural fue la que presentó mejores niveles de sostenibilidad al analizar las cuatro reservas; mientras que la dimensión económica obtuvo los niveles más bajos, a excepción del Parque Aventura San Luis, que obtuvo su menor puntaje en la dimensión administrativa, aunque este resultado no fue insatisfactorio.

De acuerdo con lo planteado por Cordero (2017), quien afirma que la sostenibilidad no se puede valorar en términos absolutos, es decir, se debe observar cualitativamente según los diferentes aspectos de cada dimensión en cada escenario particular; el plan estratégico se propuso sobre la base de los resultados puntuales de la evaluación. En relación con su implementación, el desarrollo de alianzas es un componente fundamental. Al respecto, Moya y Brenes (2017) apuntan para la misma área de estudio, que la organización local y el trabajo conjunto podrán ser claves de éxito en el desarrollo de encadenamientos. Fomentar alianzas permitirá también la mejora de las capacidades técnicas y financieras, la divulgación de información sobre las reservas y su trabajo en relación con la conservación y, finalmente, la consolidación de sus procesos a largo plazo o a perpetuidad.

Como aseguran Leung et al. (2019), las alianzas entre administradores de áreas protegidas, ONG, sociedad civil, sector privado y el Estado pueden ser muy gratificantes, pero también muy exigentes. Sin embargo, se pueden establecer alianzas eficaces entre actores interesados, si se trabaja de forma participativa para el desarrollo de planes y actividades de gestión. Lo anterior coincide con los hallazgos de Serenari et al. (2016), quienes encontraron que en Chile el desarrollo de alianzas entre las reservas privadas y universidades, ONG, agencias estatales y actores de conservación y desarrollo global, como la Organización de las Naciones Unidas, han influenciado y facilitado el establecimiento de políticas para el fortalecimiento de la conservación desde el sector privado.

\section{CONCLUSIONES}

El sistema estándar de indicadores propuesto en el modelo de estrategia de sostenibilidad permite su aplicación en cualquier reserva privada a nivel mundial, pues, aunque fue propuesto con base en el caso de las reservas analizadas en el cantón San Ramón, se abordaron aspectos presentes en cualquier reserva. De igual manera, permite excluir o incluir los indicadores que se consideren necesarios.

En cuanto a la sostenibilidad de las dimensiones analizadas, en el presente estudio la dimensión sociocultural fue la que presentó mejores niveles, al analizar las cuatro reservas; mientras que la dimensión económica obtuvo los niveles más bajos. Aunque el resultado final de la aplicación del sistema de indicadores es un porcentaje general de sostenibilidad, este valor no es suficiente para proponer acciones de mejoras concretas, que permitan fortalecer los aspectos que así lo requieran. Para esto es necesario desarrollar un análisis desde las cuatro dimensiones y, más específicamente, desde los resultados de los indicadores evaluados.

Finalmente, la implementación del plan estratégico propuesto permitirá mejorar la sostenibilidad en las reservas naturales privadas del cantón, de acuerdo con la evaluación realizada; lo cual aportará 
al fortalecimiento de la participación ciudadana, la prosperidad económica y la conservación, como bases del desarrollo sostenible. La generación de alianzas entre el sector privado, el estatal y la sociedad civil es clave para una consecución más efectiva de las acciones propuestas.

\section{REFERENCIAS BIBLIOGRÁFICAS}

Arguedas, S. (2015). Herramienta MAPE. Guía para el diagnóstico y mejoramiento de la gestión de Reservas Naturales Privadas de la Argentina. Fundación Vida Silvestre Argentina. https://bit.ly/3pVoDPi

Banco Central de Costa Rica. (2016). Cuentas ambientales de Costa Rica: Cuenta de bosque 20112013. https://bit.ly/36RJhlm

Barrientos, E., Villalobos, H. y Rodríguez, J. (2019). Propuesta de creación de la Red de Reservas Naturales del Corredor Biológico Montes de Aguacate. (Reporte Técnico). Fundación Bosque Nuboso de Occidente y Programa de Pequeñas Donaciones del Fonde Mundial para Medio Ambiente. https://bit.ly/3q92YD2

Bingham, H., Fitzsimons, J. A., Redford, K. H., Mitchell, B. A., Bezaury-Creel, J. y Cumming, T. L. (2017). Privately protected areas: advances and challenges in guidance, policy and documentation. Parks, 23(1), 13-28. http://doi.org/10.2305/IUCN.CH.2017.PARKS-231HB.en

Cifuentes, M., Izurieta, A. y Henrique, H. (2000). Measuring protected area management effectiveness. WWF Centroamérica, IUCN y GTZ. https://bit.ly/39TA6Jq

Cordero, J.C. (2017). Propuesta de un sistema de indicadores de sostenibilidad turística para destinos urbanos. Ara: Journal of Tourism Research, 7(1), 41-51. https://cutt.ly/bkMtReL

de Albuquerque, F. S., Benito, B., Beier, P., Assuncão-Albuquerque, M. J. y Cayuela, L. (2015). Supporting underrepresented forests in Mesoamerica. Natureza \& Conservação, 13(2), 152-158. https://doi.org/10.1016/i.ncon.2015.02.001

de Camino, R., Segura, O., Arias, L. G. y Pérez, I. (2000). Costa Rica. Forest strategy and the evolution of land use. The World Bank. https://bit.ly/3pOnhRL

Dinerstein, E., Olson, D., Joshi, A., Vynne, C., Burgess, N., Wikramanayake, E., Hahn, N., Palminteri, S., Hedao, P., Noss, R., Hansen, M., Locke, H., Ellis, E. C., Jones, B., Barber, C. V., Hayes, R., Kormos, C., Martin, V., Crist, E., ... Saleem, M. (2017). An ecoregion-based approach to protecting half the terrestrial realm. BioScience, 67(6), 534-545. https://doi.org/10.1093/ biosci/bix014 
Gutiérrez-Fernández, F., Cloquell, V. A. y Cloquell, V. (2012). Propuesta de un sistema de indicadores de sostenibilidad para áreas naturales con uso turístico, validado mediante consulta a terceros. Anuario Turismo y Sociedad, XIII, 55-83. https://bit.ly/2N1Pj26

Kuzek, J. y Rist, R. (2004). Manual para gestores del desarrollo. Diez pasos hacia un sistema de seguimiento y evaluación basado en resultados (Trad. M. V. Mejía). Mayo Ediciones. https://bit.ly/3cJWZRv

Langholz, J. y Lassoie, J. (2001). Combining conservation and development on private lands: Lessons from Costa Rica. Environment, Development and Sustainability, 3, 309-322. https://doi.org/10.1023/A:1020828610847

Leung, Y.-F., Spenceley, A., Hvenegaard, G. y Buckley, R. (Eds.). (2019). Gestión del turismo y de los visitantes en áreas protegidas: directrices para la sostenibilidad (Serie Directrices sobre Buenas Prácticas en Áreas Protegidas no. 27). Unión Internacional para la Conservación de la Naturaleza. https://bit.ly/39WDNhw

Montaño, M., Campos, J. J. y Louman, B. (Eds.). (2006). Uso de principios, criterios e indicadores para monitorear y evaluar las acciones y efectos de políticas en el manejo de los recursos naturales (Serie técnica no. 32). Departamento de Recursos Naturales y Ambiente, Centro Agronómico Tropical de Investigación y Enseñanza. https://bit.ly/2MJ61Uf

Moya, M.M. y Brenes, L. (2017). Rutas turísticas en la zona de amortiguamiento de la Reserva Biológica Alberto Manuel Brenes (ReBAMB). Revista Pensamiento Actual, 17(1), 1-10. https://bit.ly/2OeHN4E

Pavlotzky, B. (Comp.). (2019). Informe anual estadísticas SEMEC 2018: SINAC en números. Sistema Nacional de Áreas de Conservación. https://bit.ly/3rtuli6

Pellin, A. y Lima, V. E. (2016). Voluntary preservation on private land in Brazil: characterisation and assessment of the effectiveness of managing private reserves of natural heritage. Brazilian Geographical Journal: Geosciences and Humanities research medium, 7(1-2), 33-52. https://bit.ly/3inYDtn

Perry, C. (1998). Processes of a case study methodology for postgraduate research in marketing. European Journal of Marketing, 32(9/10), 785-802. https://doi. org/10.1108/03090569810232237

Programa Estado de la Nación. (2016). Estado de la Nación en desarrollo humano sostenible [2016]. (Serie Informe Estado de la Nación, 22-2016). PEN-CONARE. https://bit.ly/3oTEvAf 
Sánchez, O., Salazar, A. F., Thowinson, J., López, J. M. y Villareal, S. (2018). Guía para la construcción y análisis de indicadores. Departamento Nacional de Planeación de Colombia. https://bit. $\underline{\mathrm{y} / 3 \mathrm{pYWzKJ}}$

Sánchez-Azofeifa, A. (2015). Análisis de la cobertura forestal de Costa Rica entre 1960 y 2013. Ambientico: Revista mensual sobre la actualidad ambiental, (253), 4-11. https://bit. Iy/3pYqJOa

Serenari, C., Peterson, M. N., Wallace, T. y Stowhas, P. (2016). Private protected areas, ecotourism development and impacts on local people's well-being: a review from case studies in Southern Chile. Journal of sustainable tourism, 25(12), 1792-1810. https://doi.org/10.10 $\underline{80 / 09669582.2016 .1178755}$

Shumba, T., De Vos, A., Biggs, R., Esler, K. J., Ament, J. M. y Clements, H. S. (2020). Effectiveness of private land conservation areas in maintaining natural land cover and biodiversity intactness. Global Ecology and Conservation, 22, e00935. https://doi.org/10.1016/i. gecco.2020.e00935

Stolton, S. y Dudley, N. (2015). Private governance of protected areas in Africa: Cases studies, lessons learnt and conditions of success. (Study no. 2: in the series on governance of protected areas in Africa). Equilibrium Research \& IIED. https://bit.ly/39VtRVt

Anexo 1. Matriz de criterios de evaluación de sostenibilidad para las reservas analizadas

\begin{tabular}{|c|c|c|c|c|c|c|c|c|}
\hline \multirow{2}{*}{$\begin{array}{l}\text { Dimensiones de la } \\
\text { sostenibilidad }\end{array}$} & \multicolumn{5}{|c|}{$\begin{array}{l}\text { Escala de evaluación } \\
\text { de indicadores }\end{array}$} & \multirow{2}{*}{$\begin{array}{c}\text { Óptimo total } \\
\text { (\# indicadores*4) }\end{array}$} & \multirow{2}{*}{$\begin{array}{c}\text { Total } \\
\text { alcanzado }\end{array}$} & \multirow{2}{*}{$\begin{array}{c}\% \\
\text { óptimo } \\
\text { total }\end{array}$} \\
\hline & 0 & 1 & 2 & 3 & 4 & & & \\
\hline \multicolumn{9}{|c|}{ Dimensión ambiental } \\
\hline $\begin{array}{l}\text { 1.Apoyo y participación } \\
\text { comunitaria. }\end{array}$ & & & & & & \multirow{5}{*}{20} & & \\
\hline 2.Apoyo interinstitucional. & & & & & & & & \\
\hline $\begin{array}{l}\text { 3.Desarrollo de } \\
\text { investigaciones. }\end{array}$ & & & & & & & & \\
\hline $\begin{array}{l}\text { 4.Aplicación de } \\
\text { conocimientos } \\
\text { tradicionales. }\end{array}$ & & & & & & & & \\
\hline $\begin{array}{l}\text { 5.Beneficios económicos a } \\
\text { las comunidades aledañas. }\end{array}$ & & & & & & & & \\
\hline
\end{tabular}




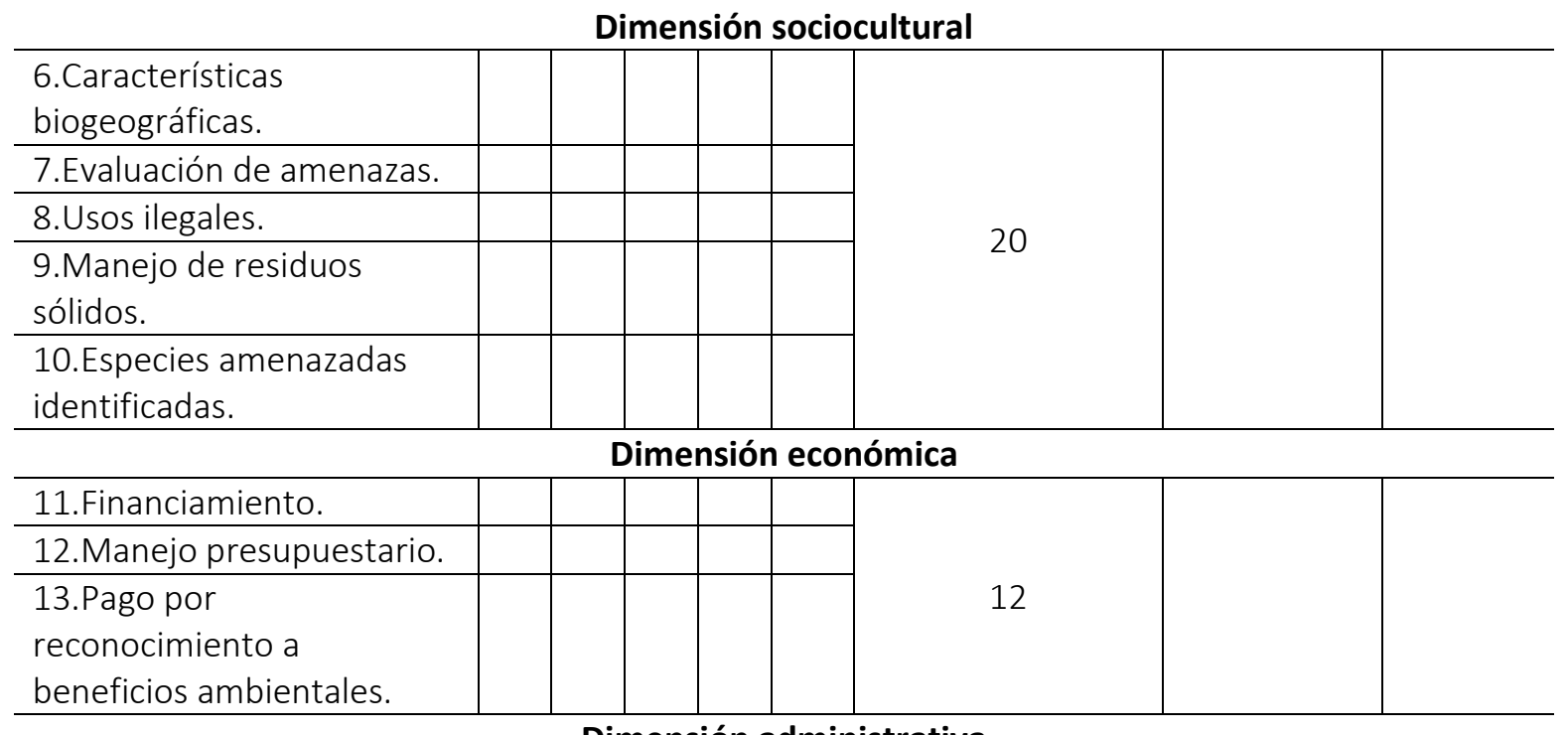

\begin{tabular}{l|l|l|l|l|l|l|l|l}
\hline \multicolumn{7}{c}{ Dimensión administrativa } & & \\
\hline $\begin{array}{l}\text { 14.Existencia de plan de } \\
\text { trabajo. }\end{array}$ & & & & & & & \\
\hline $\begin{array}{l}\text { 15.Establecimiento de } \\
\text { objetivos de conservación. }\end{array}$ & & & & & & & \\
\hline 16.Personal. & & & & & & & \\
\hline 17.Infraestructura. & & & & & & & \\
\hline $\begin{array}{l}\text { 18.Leyes y normas } \\
\text { generales. }\end{array}$ & & & & & & & \\
\hline $\begin{array}{l}\text { 19.Sistema de organización } \\
\text { de información. }\end{array}$ & & & & & & & & \\
\hline $\begin{array}{l}\text { 20.Motivación del } \\
\text { propietario para conservar. }\end{array}$ & & & & & & & & \\
\hline $\begin{array}{l}\text { 21.Aseguramiento del } \\
\text { esfuerzo de conservación } \\
\text { transgeneracional. }\end{array}$ & & & & & & & & \\
\hline
\end{tabular}

\begin{tabular}{cc|}
\hline & Escala de sostenibilidad \\
\hline Significado & \% del óptimo \\
\hline Muy satisfactorio & $91-100$ \\
Satisfactorio & $76-90$ \\
Medianamente satisfactorio & $51-75$ \\
Poco satisfactorio & $36-50$ \\
Insatisfactorio & $<35$ \\
\hline
\end{tabular}

Fuente: Elaboración propia.

\section{AGRADECIMIENTOS}

Los autores expresan su agradecimiento a los propietarios de las reservas naturales privadas, a la Fundación Bosque Nuboso de Occidente y a los expertos participantes en el proceso de investigación, por el apoyo brindado al desarrollo de la misma. 